ORIGINAL ARTICLE

\title{
Neonatal resuscitation 1: a model to measure inspired and expired tidal volumes and assess leakage at the face mask
}

\author{
C P F O'Donnell, C O F Kamlin, P G Davis, C J Morley
}

Arch Dis Child Fetal Neonatal Ed 2005;90:F388-F391. doi: 10.1136/adc.2004.064683

See end of article for authors' affiliations

Correspondence to:

Correspondence to:
Dr O'Donnell, Royal Women's Hospital

Melbourne 132 Grattan

Street, Carlton, Victoria

3053, Australia;

colm.odonnell@

rwh.org.au

Accepted 18 March 2005

Published Online First

4 May 2005

\begin{abstract}
Background: Neonatal resuscitation is a common and important intervention, and adequate ventilation is the key to success. In the delivery room, positive pressure ventilation is given with manual ventilation devices using face masks. Mannequins are widely used to teach and practise this technique. During both simulated and real neonatal resuscitation, chest excursion is used to assess tidal volume delivery, and leakage from the mask is not measured.

Objective: To describe a system that allows measurement of mask leakage and estimation of tidal volume delivery.

Methods: Respiratory function monitors, a modified resuscitation mannequin, and a computer were used to measure leakage from the mask and to assess tidal volume delivery in a model of neonatal resuscitation. Results: The volume of gas passing through a flow sensor was measured at the face mask. This was a good estimate of the tidal volume entering and leaving the lung in this model. Gas leakage between the mask and mannequin was also measured. This occurred principally during inflation, although gas leakage during deflation was seen when the total leakage was large. A volume of gas that distended the mask but did not enter the lung was also measured.

Conclusion: This system can be used to assess the effectiveness of positive pressure ventilation given using a face mask during simulated neonatal resuscitation. It could be useful for teaching neonatal resuscitation and assessing ventilation through a face mask.
\end{abstract}

r nternationally agreed consensus statements on neonatal resuscitation advise that the key to success is adequate ventilation. ${ }^{12}$ It is recommended that breathing be assisted by giving positive pressure ventilation (PPV) with a manual ventilation device via a face mask. ${ }^{12}$ Observation of chest wall movement is recommended to assess the adequacy of ventilation-that is, gas delivery to the lungs ${ }^{12}$-although the precision and accuracy of this assessment is not known. The technique of "bag and mask" ventilation is generally taught and practised using mannequins. Teaching and assessment of these techniques using mannequins form an integral part of neonatal life support courses, which are a mandatory part of physician, midwife, and neonatal nurse training in many countries. ${ }^{3}$

According to consensus statements, most newborn infants can be adequately ventilated with a bag and mask. ${ }^{2}$ However, the evidence to support this statement is lacking, the few studies of infants in the delivery room reporting that tidal volumes adequate for gas exchange were rarely delivered through a mask..$^{5-7}$ There are no reported studies investigating resuscitation of very preterm infants using a mask. One study has assessed face masks used for neonatal resuscitation. ${ }^{8}$ The participants in that study had little or no experience of resuscitating infants, manual ventilation devices were not used, and leakage was not measured.

Giving PPV to a mannequin through a face mask mimics practice in the delivery room in that "chest" excursion is used to assess the adequacy of ventilation, and there is no indication of the extent of the leak between the mask and face. To assess the adequacy of ventilation during resuscitation, measurement of the volume of gas entering and leaving the lungs (the tidal volume, $\mathrm{V}_{\mathrm{T}}$ ) would be ideal. This is, however, impractical unless the infant is intubated. In the absence of a direct measurement, an estimate of the tidal volume delivered during bag and mask ventilation, which was more accurate than the current "gold standard" of chest excursion, would be useful. A measure of leakage occurring at the face mask would also be useful, as large leaks will reduce the tidal volumes delivered and thus the effectiveness of PPV.

We therefore modified a resuscitation mannequin so that we could measure the volume of gas passing through the mask and the tidal volume delivered to a test lung during simulated neonatal resuscitation. We wished to calculate the volume of gas leaking at the mask and determine how well the tidal volume returning through the mask represented the tidal volume returning from the lung.

\section{MATERIALS AND METHODS}

\section{Manual ventilation devices and face masks}

The manual ventilation devices used at our hospital are the Neopuff infant resuscitator (Fisher \& Paykel Healthcare, Auckland, New Zealand) and the Laerdal infant resuscitator (Laerdal Medical, Oakleigh, Australia). The Neopuff is a T piece device that requires a compressed gas source. It has a valve on the outlet which allows a positive end expiratory pressure to be set for a given flow rate. Occlusion of this valve generates a predetermined peak inspiratory pressure. The Laerdal Infant Resuscitator is a $240 \mathrm{ml}$ silicone self inflating bag. We routinely use round silicone Laerdal face masks (Laerdal Medical) with both devices at our hospital, and used the appropriate size mask $(0 / 1)$ for the mannequin in this study.

Abbreviations: PPV, positive pressure ventilation; $V_{\text {MASK, volume of gas }}$ that, under pressure, distends the mask but does not enter the lung; $V_{T}$, tidal volume; $\mathrm{V}_{\mathrm{TE}}$ (mask), expiratory tidal volume at the mask; $\mathrm{V}_{\mathrm{T}(\text { mask), }}$ inspiratory tidal volume at the mask 


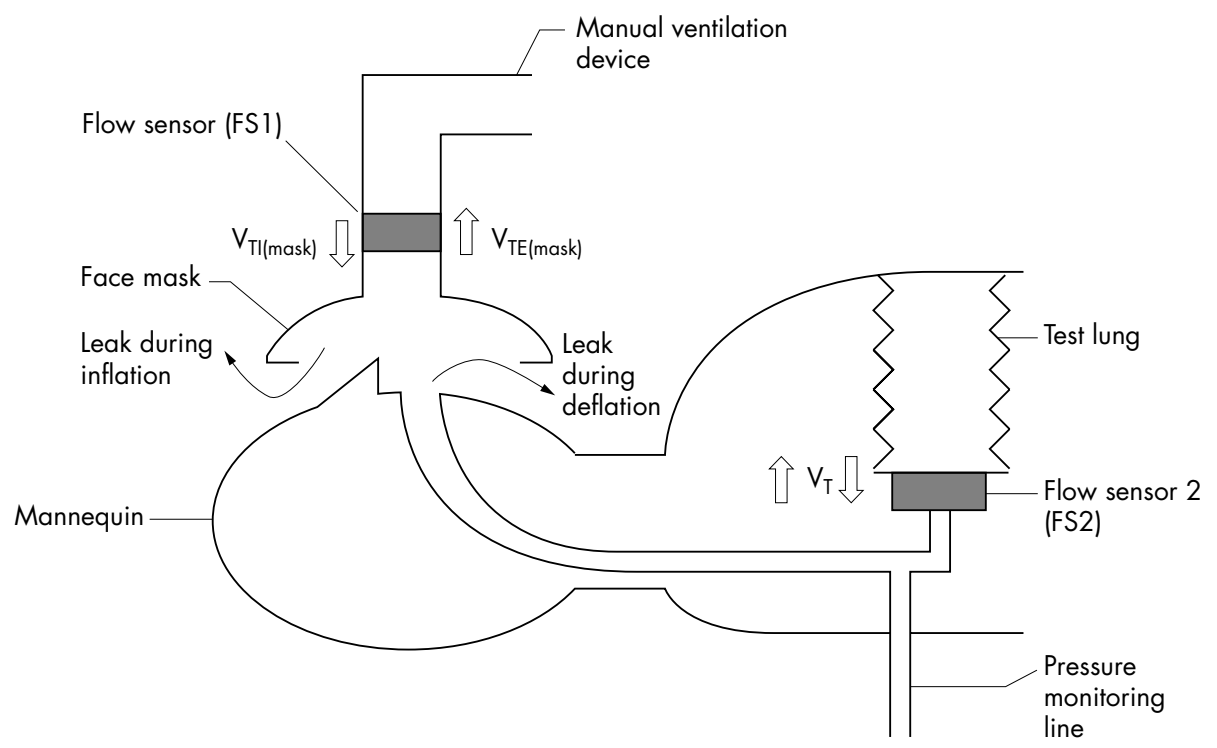

Figure 1 Diagram of apparatus used to assess accuracy of measurements made with flow sensor 1 (FS1) using flow sensor 2 (FS2). $V_{T(\text { mask), }}$

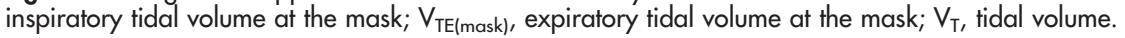

\section{Modification of the mannequin}

The Laerdal Resusci Baby mannequin (Laerdal Medical) is the most widely used resuscitation mannequin and has been found to be the most realistic for the purposes of simulated PPV. ${ }^{9}$ This mannequin is supplied with a bag in its "thorax". When PPV is given appropriately, this bag inflates and slowly deflates, causing visible "chest" rise and fall. We replaced the bag with a test lung with a baseline volume of $50 \mathrm{ml}$ (Dräger, Lubeck, Germany) and connected it via an airtight seal to the mannequin's "oropharynx", so that its inflation and deflation caused "chest" excursion similar to that of the original mannequin. A pressure monitoring line was connected immediately proximal to the test lung. The compliance of this model, calculated by measuring the inspired volume of the whole system when pressurised to $25 \mathrm{~cm} \mathrm{H}_{2} \mathrm{O}$, was

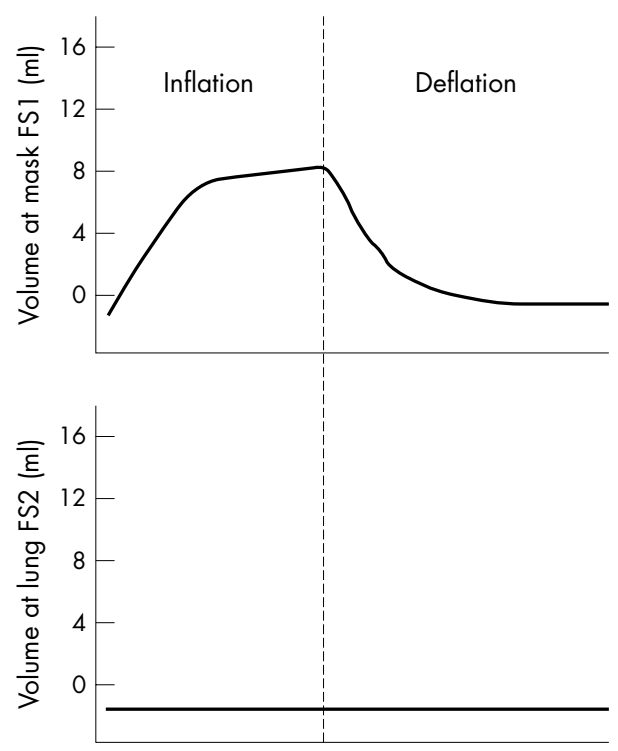

Figure 2 Trace obtained when the face mask was applied firmly to a flat bench top so that no gas escaped, and positive pressure inflation was applied. This illustrates a volume of gas distending the mask that appears to contribute to tidal volume, but never enters the lung. FS1, Flow sensor 1; FS2, flow sensor 2.
$0.46 \mathrm{~m} / \mathrm{cm} \mathrm{H}_{2} \mathrm{O}$, comparable to that of an infant with respiratory distress syndrome. ${ }^{10}$

\section{Respiratory monitor}

Two Florian Respiratory Monitors (Acutronic Medical Systems, Zug, Switzerland) were used to measure gas flow. This monitor uses a flow sensor with a hot wire anemometer with minimal $(<1 \mathrm{ml})$ dead space to detect gas flow. The monitor calculates the volumes of gas passing through the sensor by integration of the flow signal. The flow sensors from the two monitors were placed in series, and their volume measurement calibrated simultaneously using a fixed volume syringe. The monitors measure airway pressures directly and were calibrated against a column of water. The output from the Florian monitors was acquired using an analogue-digital converter using the Spectra software program (Grove Medical, London, UK). This is a computer program specifically designed for the acquisition and analysis of respiratory signals.

\section{Values measured}

We placed one flow sensor (FS1, fig 1) between the manual ventilation device and the face mask. With this sensor, we measured the volume of gas passing from the device through the mask-the inspiratory tidal volume at the mask $\left(\mathrm{V}_{\mathrm{TI}(\mathrm{mask})}\right)$-and the volume of gas returning from the mannequin through the mask-the expiratory tidal volume at the mask $\left(\mathrm{V}_{\mathrm{TE} \text { (mask) }}\right)$. We placed the second sensor (FS2, fig l) in the mannequin's "airway", proximal to the test lung. With this sensor we measured the tidal volume $\left(\mathrm{V}_{\mathrm{T}}\right)$ entering and leaving the test lung.

\section{Values calculated}

If the volume of gas passing from the device through the mask exceeded that returning from the mannequin through the mask, there was a leak between the mask and the face. This was calculated as their difference expressed as a percentage of the inspiratory tidal volume at the mask:

leak $(\%)=\left(\left(\mathrm{V}_{\mathrm{TI}(\text { mask })}-\mathrm{V}_{\mathrm{TE}(\text { mask })}\right) / \mathrm{V}_{\mathrm{TI}(\text { mask })}\right) \times 100$.

If leakage from the mask was detected, we wished to determine whether it occurred during inspiration or expiration. 
We hypothesised that if the volume of gas passing from the device through the mask $\left(\mathrm{V}_{\mathrm{TI} \text { (mask) }}\right)$ did not equal the volume returning through the mask from the mannequin $\left(\mathrm{V}_{\mathrm{TE} \text { (mask) }}\right)$ or the tidal volume measured in the mannequin $\left(\mathrm{V}_{\mathrm{T}}\right)$, there could be three potential reasons:

(1) Leakage during inflation. In the presence of a poor seal between the mask and face, some of the gas passing through FSI would escape during inflation and not pass through FS2 at the lung. The volume leaking during inflation would thus contribute to $\mathrm{V}_{\mathrm{TI} \text { (mask) }}$ but not to $\mathrm{V}_{\mathrm{T}}$ or $\mathrm{V}_{\mathrm{TE} \text { (mask). }}$.

(2) Leakage during deflation. In the presence of a poor seal between the mask and face, some of the gas leaving the lung through FS2 would escape around the mask during deflation and not pass through FS1, resulting in $\mathrm{V}_{\mathrm{TE} \text { (mask) }}$ underestimating $\mathrm{V}_{\mathrm{T}}$.

(3) Distension of the mask. The mask used is made of distensible silicone; thus we hypothesised that, in the presence of a reasonable seal between the mask and face, gas under pressure would distend the mask but not enter the lung $\left(\mathrm{V}_{\text {MASK }}\right)$. This would pass through FSI during inflation and back through FSl during deflation, thus contributing to $\mathrm{V}_{\mathrm{TI} \text { (mask) }}$ and $\mathrm{V}_{\mathrm{TE} \text { (mask) }}$ but not to $\mathrm{V}_{\mathrm{T}}$. This volume was demonstrated by placing the mask flat on a bench top and giving positive pressure inflations (fig 2).

To test this system, we recorded 100 inflations, applied by a consultant neonatologist, with various amounts of leakage at the face mask, with each manual ventilation device. Inflations with a peak inspiratory pressure of $25 \mathrm{~cm} \mathrm{H}_{2} \mathrm{O}$ and positive end expiratory pressure $5 \mathrm{~cm} \mathrm{H}_{2} \mathrm{O}$ were given with the Neopuff. With the Laerdal bag a manometer was placed in the circuit and a peak inspiratory pressure of $25 \mathrm{~cm} \mathrm{H}_{2} \mathrm{O}$ was targeted for inflations. Data were analysed using SPSS for Windows (SPSS Inc, Chicago Illinois, USA). Results are expressed as mean (SD).

\section{RESULTS}

The mannequin's airway and lung were leak free, with a difference between the volume entering and leaving the lung of $-0.2(0.5) \%$. There were no significant differences in the $\mathrm{V}_{\mathrm{TI} \text { (mask), }} \mathrm{V}_{\mathrm{TE} \text { (mask), }}$ or $\mathrm{V}_{\mathrm{T}}$ delivered with each manual ventilation device.

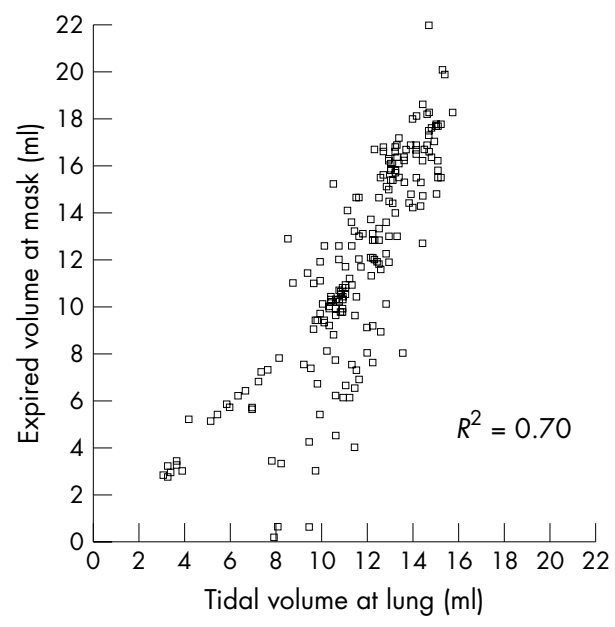

Figure 3 Scatter plot showing the relation of the expired tidal volume at the mask to the tidal volume at the lung.
Relationship of $V_{T E \text { (mask) }}$ to $V_{T}$

The volume returning from the mannequin through the mask $\left(\mathrm{V}_{\mathrm{TE} \text { (mask) }}\right)$ was a good estimate of the volume leaving the lung $\left(\mathrm{V}_{\mathrm{T}}\right)$ (fig 3$)$. When there were large leaks $(>51 \%)$, $\mathrm{V}_{\mathrm{TE}(\text { mask) }}$ tended to underestimate $\mathrm{V}_{\mathrm{T}}$ because some of the gas returning from the lung escaped around the mask during deflation (see under Leak). With smaller leaks, $\mathrm{V}_{\mathrm{TE} \text { (mask) }}$ tended to overestimate $V_{T}$ because a volume of gas distended the mask but did not enter the lung (see under Volume distending the mask and not entering the lung $\left.\left(\mathrm{V}_{\mathrm{MASK}}\right)\right)$. Overall, $\mathrm{V}_{\mathrm{TE} \text { (mask) }}$ was $100.6(24.8) \%$ of the lung $\mathrm{V}_{\mathrm{T}}$.

\section{Leak}

If the volume of gas passing from the ventilation device through the mask was greater than that returning from the mannequin through the mask-that is, $\mathrm{V}_{\mathrm{TI}(\text { mask) }}>$

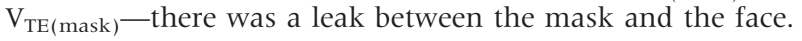
When the volume returning from mannequin through the mask $\left(\mathrm{V}_{\mathrm{TE} \text { (mask) }}\right)$ was greater than or equal to the volume leaving the lung $\left(\mathrm{V}_{\mathrm{T}}\right)$, all of the leak occurred during inflation.

When the volume leaving the lung $\left(\mathrm{V}_{\mathrm{T}}\right)$ was greater than the volume returning from the mannequin through the mask $\left(\mathrm{V}_{\mathrm{TE}(\text { mask })}\right)$, a proportion of the leak occurred in deflation. Leaks during deflation were never seen without a leak during inflation and only seen when total leakage was greater than $51 \%$. When there was a large leak, the proportion occurring during inflation $\left(\left(\left(\mathrm{V}_{\mathrm{TI}(\text { mask })}-\mathrm{V}_{\mathrm{T}}\right) /\left(\mathrm{V}_{\mathrm{TI}(\text { mask })}-\mathrm{V}_{\mathrm{TE}(\text { mask })}\right)\right) \times\right.$ $100)$ was $89(10) \%$, and the proportion during deflation $\left(\left(\left(\mathrm{V}_{\mathrm{T}}\right.\right.\right.$ $\left.\left.\left.-\mathrm{V}_{\mathrm{TE}(\text { mask })}\right) /\left(\mathrm{V}_{\mathrm{TI}(\text { mask })}-\mathrm{V}_{\mathrm{TE}(\text { mask })}\right)\right) \times 100\right)$ was $11(10) \%$.

\section{Volume distending the mask and not entering the lung ( $\mathrm{V}_{\text {MASK }}$ )}

When the volume passing from the device through the mask $\left(\mathrm{V}_{\mathrm{TI}(\text { mask })}\right)$ equalled the volume returning from the lung $\left(\mathrm{V}_{\mathrm{TE} \text { (mask) }}\right)$, there was no leakage from the mask. During these inflations, $\mathrm{V}_{\text {MASK }}$ was readily demonstrated, as the volume passing through FSI was greater than that passing through FS2 and entering the lung. Although this became progressively smaller as the total leak increased, $\mathrm{V}_{\text {MASK }}$ was seen with leaks up to $51 \%$. This volume was $2.4(1.4) \mathrm{ml}$, which represented $18.3(10.5) \%$ of $\mathrm{V}_{\mathrm{T}}$.

\section{DISCUSSION}

Positive pressure ventilation using manual ventilation devices via a face mask is an important skill. It is widely taught and practised using mannequins. We have developed a system that allows estimation of tidal volume delivered and measurement of mask leak during simulated PPV. The pressures used may also be recorded using this system. This system was developed with a view to recording real resuscitations in the delivery room.

Studies of PPV through a mask during resuscitation in the delivery room are few. In a study of term infants, "bag and mask" ventilation seemed relatively inefficient, with tidal exchange substantially less than that seen after intubation and rarely sufficient to produce adequate alveolar ventilation. ${ }^{5}$ It is worth noting that the apparatus used to measure expired tidal volumes in this ${ }^{5}$ and other assessments of bag and mask ventilation ${ }^{67}$ was similar to ours, in that the flow sensor (pneumotachograph) was placed between the manual ventilation device and mask. Although the mask used for these studies differed from ours and its properties are probably not identical, there was leakage from this mask during inflation. It is thus reasonable to speculate that there may also have been leakage during expiration. It is unclear how the expired tidal volume reported (equivalent to our $\mathrm{V}_{\mathrm{TE}(\mathrm{mask})}$ ) related to the expired tidal volume leaving the lungs. 


\section{What is already known on this topic}

- Resuscitation mannequins are used to teach and practise "bag and mask" ventilation techniques

- During mask ventilation in both simulated and real neonatal resuscitation, there is no indication of the extent of leakage from the mask, and chest wall movement is used to assess the adequacy of ventilation

\section{What this study adds}

- We have adapted the most commonly used mannequin to allow measurement of leakage from masks and estimation of tidal volume delivered to the lungs

- The volume of gas returning through the mask is a good estimate of the volume leaving the lungs in this model

It remains unclear how effective bag and mask ventilation in the delivery room is as a means of delivering a satisfactory tidal volume, particularly in preterm infants, for which there are no reported studies. Moreover, this technique is not without its complications; bradycardia caused by application of a mask during neonatal resuscitation has been reported. ${ }^{11}{ }^{12}$ Detailed studies of the efficacy of PPV through a face mask in newborn infants are needed, particularly in preterm infants. Applying a flow sensor between the manual ventilation device and face mask in the delivery room may give valuable insights into the effect of PPV on newborn infants during resuscitation.

\section{CONCLUSIONS}

We have developed a system for practising and assessing PPV using a face mask. In this model, the volume of gas returning through the face mask from the mannequin is a good estimate of the tidal volume entering and leaving the lung. Most leakage from face masks occurs during inflation, although a small proportion does occur during deflation when the total leakage is large $(>51 \%)$. With leaks of up to $51 \%$, a volume of gas distends the mask but does not enter the lungs. These data will be useful for further evaluation of resuscitation equipment and techniques in bench top studies and in the delivery room.

\section{ACKNOWLEDGEMENTS}

CPFO'D is in receipt of the Royal Women's Hospital Postgraduate Degree Scholarship. PGD is supported by a National Health and Medical Research Council Fellowship. We thank Dr Brendan Murphy and an anonymous reviewer for helpful comments that have considerably improved this paper.

\section{Authors' affiliations}

C P F O'Donnell, C O F Kamlin, P G Davis, C J Morley, Royal Women's Hospital Melbourne, Victoria 3053, Australia

Competing interests: none declared

\section{REFERENCES}

1 Kattwinkel J, Niermeyer S, Nadkarni V, et al. An Advisory Statement From the Pediatric Working Group of the International Liaison Committee on Resuscitation. Pediatrics 1999;103:e56.

2 Contributors and Reviewers for the Neonatal Resuscitation Guidelines. International Guidelines for Neonatal Resuscitation: an excerpt from the Guidelines 2000 for Cardiopulmonary Resuscitation and Emergency Cardiovascular Care: International Consensus on Science. Pediatrics 2000; 106:e29.

3 Kattwinkel J, ed. Neonatal resuscitation program: textbook of neonatal resuscitation, 4th ed. Washington DC: American Academy of Pediatrics/ American Heart Association, 2000.

4 Richmond S, ed. Resuscitation at birth: the newborn life support provider manual. London: Newborn Life Support Working Party, Resuscitation Council (UK), 2001

5 Milner AD, Vyas $\mathrm{H}$, Hopkin IE. Efficacy of facemask resuscitation at birth. BMJ 1984;289:1563-5.

6 Field D, Milner AD, Hopkin IE. Efficiency of manual resucitators at birth. Arch Dis Child 1986;61:300-2.

7 Hoskyns EW, Milner AD, Hopkin IE. A simple method of face mask resuscitation at birth. Arch Dis Child 1987;62:376-8.

8 Palme C, Nystrom B, Tunell R. An evaluation of the efficiency of face masks in the resuscitation of newborn infants. Lancet 1985;1:207-10.

9 Howells R, Madar J. Newborn resuscitation training: which manikin. Resuscitation 2002;54:175-81.

10 Morley CJ, Greenough A. Respiratory compliance in premature babies treated with artificial surfactant (ALEC). Arch Dis Child 1991;66:467-71.

11 Finer NN, Rich W. Neonatal resuscitation: toward improved performance. Resuscitation 2002;53:47-51.

12 Sinclair JC, Bracken MB, eds. Effective care of the newborn infant. Oxford: Oxford University Press, 1999:30. 